Research Article

\title{
Controlling Conical Beam Carrying Orbital Angular Momentum with Transmissive Metasurface
}

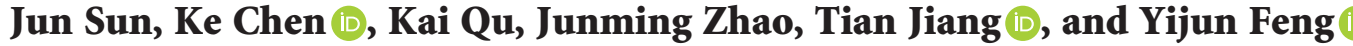 \\ School of Electronic Science and Engineering, Nanjing University, Nanjing 210093, China \\ Correspondence should be addressed to Ke Chen; ke.chen@nju.edu.cn and Yijun Feng; yjfeng@nju.edu.cn
}

Received 14 March 2021; Revised 30 April 2021; Accepted 22 May 2021; Published 30 May 2021

Academic Editor: Flaminio Ferrara

Copyright (c 2021 Jun Sun et al. This is an open access article distributed under the Creative Commons Attribution License, which permits unrestricted use, distribution, and reproduction in any medium, provided the original work is properly cited.

Conical beams have potential uses in wireless and satellite-based communication. In this study, we propose a method using a transmissive metasurface to achieve full control of the diverging effect of orbital angular momentum (OAM) modes to form the desired conical beam. A patch antenna functioning as the feed source is combined with the transmissive metasurface to enable the integration of the source and metasurface. For full control of conical radiation, including the cone angle and OAM mode, we introduce both radial and circumferential phase gradients to the proposed metasurface. Experiments are conducted in the microwave region to validate the design method, which shows good agreement with the simulation results. The proposed metasurface provides a means of flexibly generating conical beams with the designed OAM mode to assist potential applications in high-speed wireless communication.

\section{Introduction}

A conical beam, which has an omnidirectional far-field pattern in the azimuth plane with the main lobe tilted to a specific angle in the elevation plane [1-3], has recently attracted considerable attention for its use in satellite communications. The characteristics of conical radiation can ensure that the terminal devices of moving vehicles and airplanes have access to satellite signals with stable performance. Compared with other methods (e.g., a phasedarray antenna with a dynamic beam to track satellite signals), conical beams are more cost-effective and convenient for fabrication without requiring a complex phase-shifting network. Therefore, several methods have been proposed for generating conical beams in the microwave region using a circular-polarized patch antenna [2], periodic microstrip radial array antenna [4], or multiring array antennas [5].

Metasurfaces, which are types of artificially engineered materials composed of subwavelength-scaled building blocks [6,7], have recently been implemented in many realworld applications. By introducing phase discontinuities across the interface, metasurfaces effectively provide unprecedented abilities in manipulating the amplitude, phase, and polarization of electromagnetic waves from the microwave region to visible light. As a result, they offer a new platform for designing flat-profile and high-efficiency functional devices such as ultrathin metalenses $[8,9]$, lowscattering surfaces [10], holographic imagers [11], and many other devices that are difficult or even impossible to fabricate using natural materials [12-18]. One of the most important applications of metasurfaces is to generate arbitrarily desired orbital angular momentum (OAM) vortex waves within a small fractional wavelength. OAM beams can provide new degrees of freedom (DOFs) to improve considerably the data capacity of communication systems and possibly solve the problems of increasingly large wireless data link budgets [19-27]. However, some limitations exist in using OAM modes for increasing channel capacity [28, 29], for example, in long-range communication links. The OAM may have useful applications in manipulating the radiation patterns of an antenna system or improving the capabilities of increasing the performance of radar systems [30-32]. An OAM beam naturally has a conical far-field pattern due to its singular point in the beam center, and by synthesizing the constant phase gradient in both the circumferential and radial directions, our previous work showed that free 
combinations of the cone angle and OAM mode can be achieved in the far-field region [21] using a reflective metasurface under the illumination of a circularly polarized plane wave. However, transmission operations are more conducive to some applications because reflective metasurfaces often have the problem of feed blockage.

In this study, we propose a transmissive metasurface for controlling the diverging effect of the OAM mode to enable full manipulation of the conical beam characteristics for linearly polarized waves. Cascaded structural configuration of meta-atoms is employed to enable high transmission amplitude and full convergence of the transmission phase. In particular, the metasurface is codesigned with a feed source of the patch antenna, enabling the integration of the source and metasurface for real-world applications. By controlling the radial phase gradient and OAM modes, we show that the transmissive metasurface can produce conical beams with desired cone angles. For verification, experiments are conducted in the microwave region, and the measured results prove to be in good agreement with the simulated results. The proposed transmissive metasurface has the advantages of having a low profile and being light weight without the problem of feed blockage that typically exists in reflective (e.g., reflect array) applications.

\section{Element Design}

Figure 1 shows schematically the wave functionality of the proposed metasurface. When the primary propagating wave from the primary feed source imposes on the metasurface, additional spatial phases are imparted to the incidence by the designed metasurface, reshaping the output into a helical wavefront-carrying predefined OAM mode, which produces a conical beam in the far-field region. Each element of the metasurface operates as a phase shifter, which can be designed with high transmission efficiency and a desired phase-tuning function. Because the overall wavefront is the collective result of the secondary radiation from each individual element illuminated by the primary source, we must design the phase pattern on the metasurface aperture with spatially varying distribution. More specifically, the phase pattern should first compensate for the out-of-phase wavefront radiated from the feed source because the patch antenna has an electrically small aperture size, naturally radiating a low-directive beam with a quasi-spherical wavefront. The proposed method can be described as one that uses a metasurface containing both radial and circumferential phase gradients (i.e., OAM mode) to engineer the cone angle of the conical radiation beam.

To determine the properties of conical beam generation, we can refer to the phased-array antenna theory and regard each metasurface element as a type of electromagnetic scatter with a predefined initial phase [25]. If we assume a cylindrical coordinate, the elevation angle and azimuth angle are denoted by $\theta$ and $\varphi$, respectively. The cone angle, which is one of the most important parameters of the conical beam, is defined by the angle between the maximum radiation direction and the $z$ axis in the elevation plane, which is denoted by the parameter $\alpha$. To realize full control of the conical

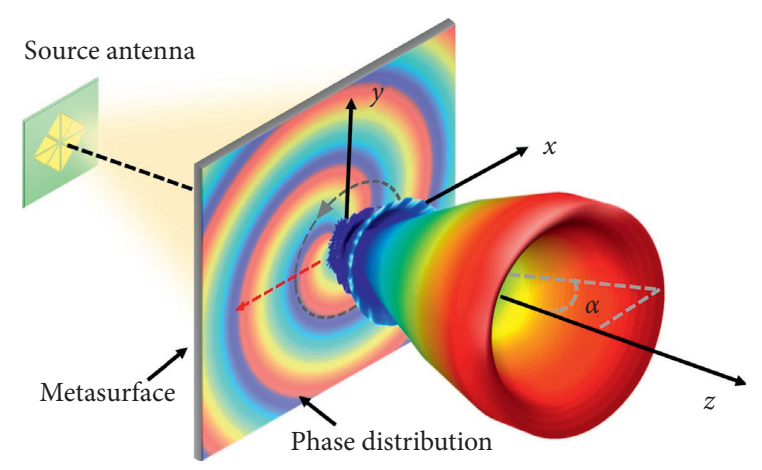

FIGURE 1: Schematic of the proposed transmissive metasurface. The primary radiation from a patch antenna is reshaped into a conical radiation when it passes through the proposed metasurface through a predesigned transmission phase pattern. The red and gray dotted lines on the metasurface denote the radial and circumferential directions, respectively.

beam generation that includes the OAM mode (denoted by the parameter $l$ ) and the cone angle $\alpha$, here we introduce a constant phase gradient in both the circumferential and radial directions to provide more flexibility and a great number of DOFs. The phase differences in the circumferential and radial directions (denoted by $\delta_{c}$ and $\delta_{r}$, respectively) are defined as the phase differences between adjacent elements in the circumferential and radial directions (Figure 1 , gray circle and red line), respectively. If we assume $a_{0}$ as the reflection phase of the element in the center of the metasurface, the reflection phase of the $m n$th element of the metasurface can be calculated as $a_{m n}=a_{0}+\delta_{c}(n-1)+\delta_{r}$ $(m-1)$. For a given metasurface aperture, the far-field pattern of the metasurface generator can be calculated theoretically using a spherical coordinate, with the elevation and azimuth angles represented by $\theta$ and $\varphi$, respectively [21, 33]:

$$
F(\theta, \phi)=\sum_{m=1}^{M} \sum_{n=1}^{N_{m}} I_{m n} e^{-i\left(k r_{m n} \sin \theta \cos \left(\phi-\phi_{m n}\right)-a_{m n}\right)},
$$

where $k$ is the phase constant in free space, $I_{m n}$ is the transmission amplitude of the $m n$th element, and $r_{m n}$ and $\varphi_{m n}$ represent the radial coordinate and azimuth angle of the $m n$th element in a spherical coordinate, respectively. In the theoretical model, $I_{m n}$ is the output transmission amplitude from the meta-atoms when the primary incidence from the feed source is considered. The method of controlling the cone angle mainly relies on the spreading effect of the OAM beam. Then, the key step to achieving a transmissive metasurface for conical beam generation is to design the element with a high transmission amplitude and wide accessible range of the transmission phase.

Several routes exist for obtaining a transmissive metasurface for high-efficiency phase-tuning operations. One is to use the Huygens' metasurface, which is typically composed of a magnetic ring structure and pair of metallic wires at microwave frequencies [34, 35]. However, Huygens' metasurface is not suitable for dual-polarization operations because of its unique structural configuration. Another method is to use cascaded metasheets, which excite both 
electric and magnetic resonances to enable high-efficiency transmission $[36,37]$. To demonstrate a metasurface capable of generating a desirable conical beam, in this study, we use a cascaded structure to implement the transmissive metasurface element, where the optimized structures are shown in Figures 2(a) and 2(d). The meta-atom is composed of four metallic patterns separated by dielectric layers. The metallic patterns are made of copper with a conductivity of $5.8 \times 10^{7} \mathrm{~S} / \mathrm{m}$, and the dielectric substrate has a thickness of $h=1.524 \mathrm{~mm}$ with a permittivity of $3.55+0.0027 i$. Two types of meta-atoms exist: one consisting of identical metallic patterns, as shown in Figure 2(a), and another consisting of two identical patterns placed in the top and bottom layers (with another pattern in the middle layers), as shown in Figure 2(d). These two types of meta-atoms have the same period $(p=5 \mathrm{~mm})$ and operate at approximately $13 \mathrm{GHz}$.

We also conduct full-wave simulations to see the electromagnetic responses of the meta-atoms using the commercial software CST Microwave Studio ${ }^{\mathrm{TM}}$. Unit-cell boundary conditions are applied in the $x$ and $y$ directions, whereas open boundary conditions are applied in the $z$ direction in free space. For meta-atoms composed of identical metallic patterns, the transmission phase can vary from approximately $40^{\circ}$ to $-190^{\circ}$ at $13 \mathrm{GHz}$ as the radius of the circular patch is changed from 0.2 to $2.4 \mathrm{~mm}$. Simultaneously, the transmission amplitude is maintained at a value higher than 0.81, as shown in Figures 3(b) and 3(c). For the other type shown in Figure 3(d), the transmission amplitude is always higher than 0.89 , whereas the transmission phase can cover a range of approximately $0^{\circ}$ to $130^{\circ}$ when the geometric parameters are changed, as shown in Figures 3(e) and 3(f). The phase curves with various geometries show a nearly linear response as a function of frequencies of approximately $13 \mathrm{GHz}$, indicating that the metasurface can also function at these frequencies. With the aforementioned two types of meta-atoms, the transmission phase can realize various phases with high transmission amplitude. Moreover, because the structures of meta-atoms have both mirror and $90^{\circ}$ rotational symmetries, meta-atoms exhibit the same electromagnetic responses for $x$ - and $y$ linearly polarized incidences without experiencing the polarization-converting effect. Here, we use a metastructure with four cascaded layers to provide a greater number of DOFs in optimizing the performance, including an increased bandwidth and improved transmission efficiency. Based on these meta-atoms, we can construct a metasurface for the generation of the conical beam carrying OAM.

\section{Simulation Results of the Conical Beam}

To verify the feasibility of the metasurface, we next describe the design process of the OAM beam generator by the proposed metasurface with a spatially varying phase in both the circumferential and radial gradients. The metasurface had a size of $200 \times 200 \mathrm{~mm}$ in the $x-y$ plane, with total pixels of $40 \times 40=1600$ elements. The incidence was produced by a patch antenna polarized in the $y$ direction. The patch antenna, as shown in Figure 3(a), was composed of three metallic layers and two dielectric spacers. The radiation part of the antenna comprised its top layer, which was shaped into a square pattern with splits, which were necessary to extend the working bandwidth. A slot was cut in the middle metallic layer, and a fraction of the transmission line was etched on the bottom metallic layer to enable a coupling feed of the antenna. The feed antenna was placed $80 \mathrm{~mm}$ from the metasurface aperture along the $z$ axis. The geometric parameters of the patch antenna were fixed as follows: $W_{1}=9.26 \mathrm{~mm}, W_{2}=25 \mathrm{~mm}, g=0.35 \mathrm{~mm}, L_{s}=12.2 \mathrm{~mm}$, $W_{s}=0.65 \mathrm{~mm}, L_{f}=12.2 \mathrm{~mm}$, and $W_{f}=1 \mathrm{~mm}$. The thicknesses of the first and second dielectric substrates were 1.524 and $0.508 \mathrm{~mm}$, respectively.

The phase distribution on the metasurface aperture from the primary feed source was calculated by assuming the feed source as the point source. However, this method is typically used for a field observed far away from the source. Note that this scenario will produce some calculation errors in phase distribution. Thus, we straightforwardly simulated it using full-wave calculation software and mapped the field where the metasurface was placed. The continuous phase profile on the metasurface illuminated by the primary radiation at $13 \mathrm{GHz}$ was recorded, as shown in Figure 3(b). This continuous phase profile was discretized into pixels by considering the pixel size of the metasurface aperture, which was then compensated by a phase distribution, as shown in the right panel of Figure 3(b), to enable a planar phasefront. Accordingly, we could then use the theoretical framework developed in our previous work, where the planar phasefront of incidence was used to calculate the scattering pattern of the metasurface [21]. More specifically, each meta-atom acted as an electromagnetic radiator, collectively contributing to the far-field radiation pattern. Through this method, the radiation pattern, including the cone angle and OAM mode, could be theoretically calculated for a given phase distribution on the metasurface.

A phase distribution to carry the desired OAM mode was added to the compensated phase (Figure 3(b)), thus forming the final desired phase distribution on the metasurface aperture. Here, we use OAM mode $l=1$ as the design example to illustrate how to synthesize the conical beam. Figure 3(c) shows the final designed phase distribution on the metasurface for the vortex beam of $l=1$ without the phase gradient in the radial direction $\left(\delta_{r}=0^{\circ}\right)$. The phase function at an arbitrary position $(x, y)$ of the metasurface was then interpreted by the real meta-atom using various geometric parameters, where the configured metasurface is shown at the bottom of Figure 3(d). The secondary transmission from the meta-atoms will form a helical wavefront, leading to a conical beam in the far-field region. To validate this property, the metasurface was illuminated by a linearly polarized incident wave with an open boundary in all directions using the aforementioned commercial software, and the results are shown in the left panels of Figures 3(c) and $3(\mathrm{~d})$. The three-dimensional (3D) radiation results revealed that the maximum radiation was tilted in a direction of $\alpha=5^{\circ}$ with a zenith along the surface normal, clearly revealing conical beam radiation. The simulated cone angle was $\alpha=5^{\circ}$, 

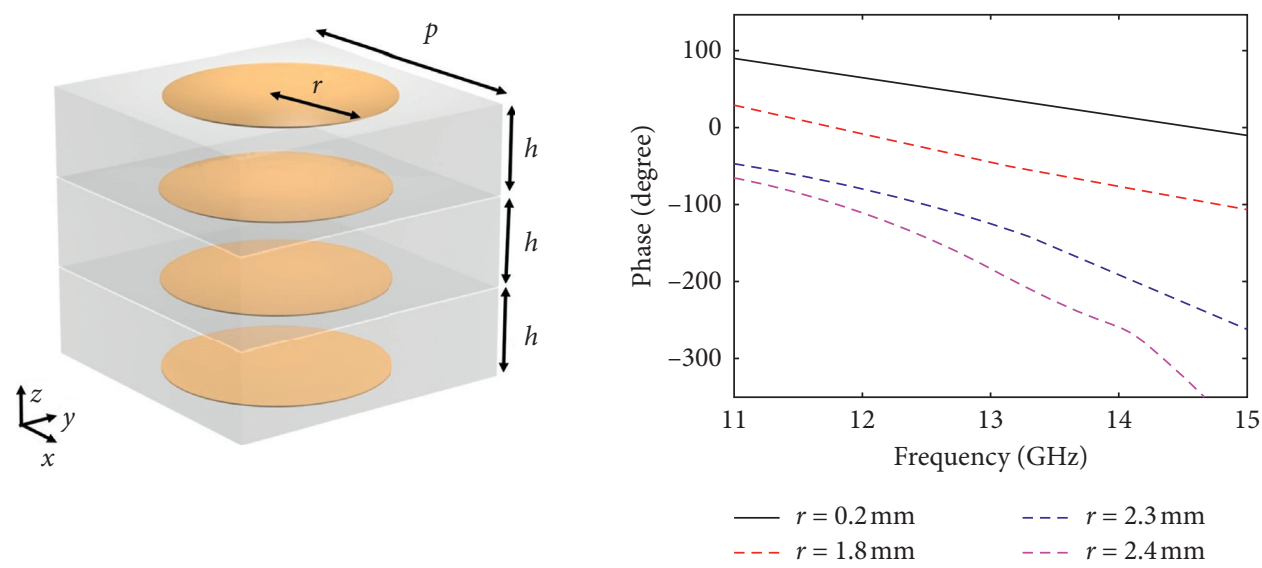

(a)

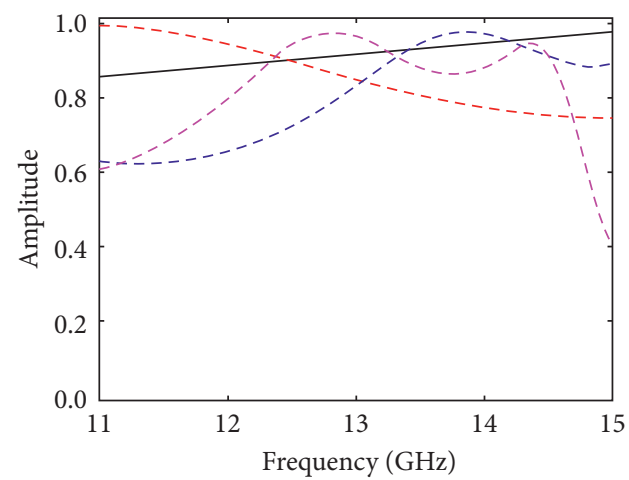

(b)

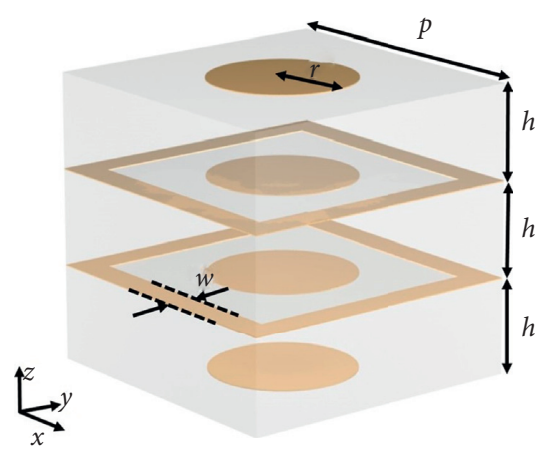
$r=0.2 \mathrm{~mm}$
- - $r=2.3 \mathrm{~mm}$
- - $r=1.8 \mathrm{~mm}$
- - $r=2.4 \mathrm{~mm}$

(c)

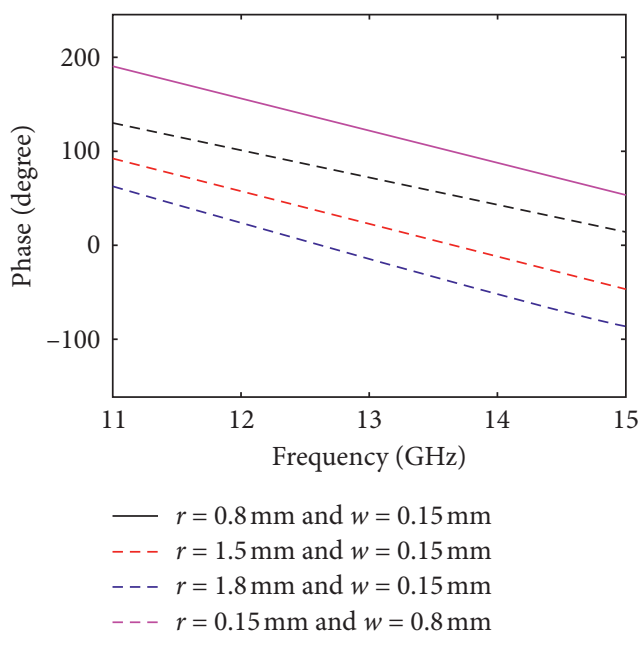

(e) (d)

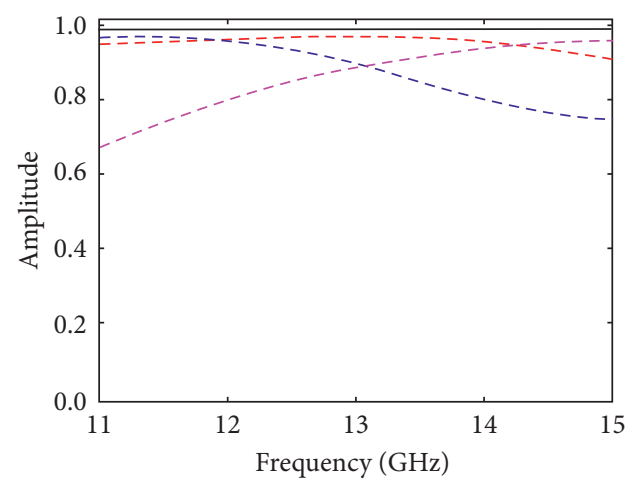

$\begin{aligned}-r & =0.8 \mathrm{~mm} \text { and } w=0.15 \mathrm{~mm} \\ ---r & =1.5 \mathrm{~mm} \text { and } w=0.15 \mathrm{~mm} \\ ---r & =1.8 \mathrm{~mm} \text { and } w=0.15 \mathrm{~mm} \\ ---r & =0.15 \mathrm{~mm} \text { and } w=0.8 \mathrm{~mm}\end{aligned}$

(f)

Figure 2: (a) Schematic of the proposed meta-atom composed of identical metallic patterns, and its (b) transmission phase and (c) transmission amplitude as functions of geometric parameters and frequencies. (d) Schematic of the proposed meta-atom composed of different metallic patterns, and its (e) transmission phase and (f) transmission amplitude as functions of geometric parameters and frequencies. 


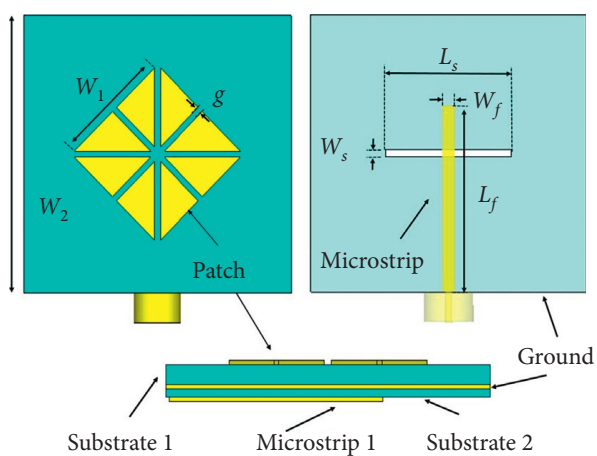

(a)
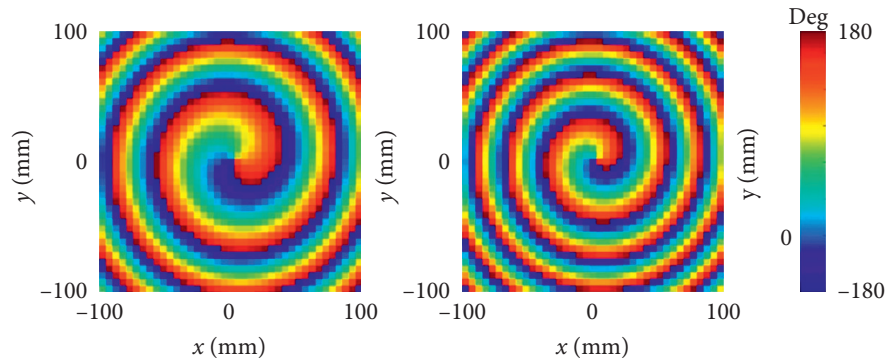
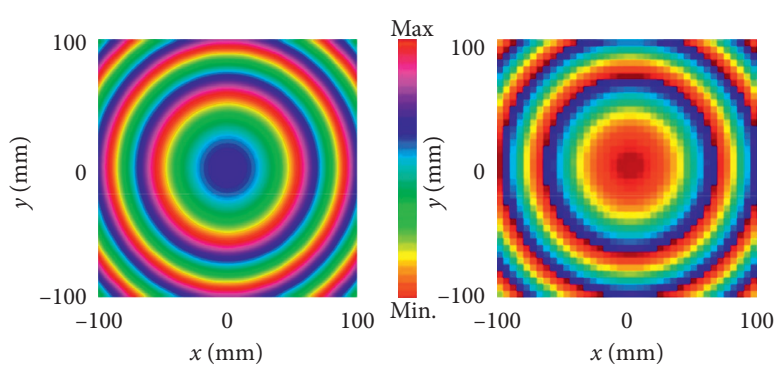

(b)
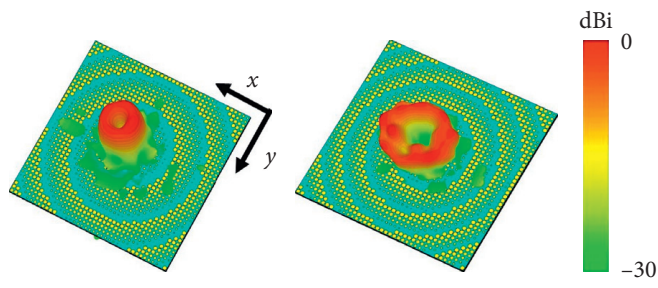

(d)
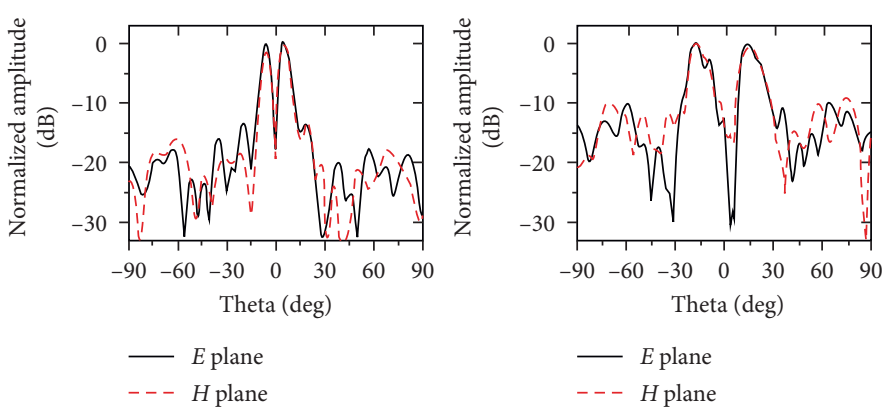

(e)

Figure 3: (a) Schematic of the feed antenna. The middle metallic pattern has a slot cut in it with a length of Ls $=12.2 \mathrm{~mm}$ and width of Ws $=0.65 \mathrm{~mm}$. (b) Continuous phase profile of the primary feed antenna illuminating the metasurface (left panel), and phase distribution used to compensate the out-of-phase incidence (right panel). (c) Transmission phase function of the metasurface. (d) 3D simulated far-field radiation pattern. (e) $2 \mathrm{D}$ simulated results in the $E$ and $H$ planes for OAM $l=1$ at $\delta_{r}=0^{\circ}$ (left panels) and $\delta_{r}=15^{\circ}$ (right panels).

which showed good agreement with the theoretical value of $\alpha=5^{\circ}$. The $2 \mathrm{D}$ radiation pattern in the $E$ and $H$ planes also verified the conical property.

Introducing a constant phase gradient in the radial direction can provide a greater number of DOFs in controlling the conical beam and can help in flexibly designing the cone angle. As examples, $\delta_{r}=15^{\circ}$ was applied to the phase distribution with a bare circumferential phase gradient, and the final designed phase functions by the metasurface are shown in the right panel of Figure 3(c). Open boundary conditions were applied in all directions in the full-wave simulation of the metasurface. The theoretical and simulated cone angles for $\delta_{r}=15^{\circ}$ were both $\alpha=15^{\circ}$. The 2D plots of the far-field radiation provide clear views of the radiation properties, further verifying the conical beam generation, as shown in Figure 3(e). The aforementioned results reveal that the cone angle of a conical beam can be flexibly tuned by employing different radial phase gradients. The cone angle is not limited to that simulated here, and in principle, many other cone angles can be realized because the transmission phase of the metasurface can be arbitrarily designed.

The OAM mode of the generated conical beam can also be flexibly changed simply by arranging different phase gradients in the circumferential direction. As a design example, we showed that OAM modes of $l=2$ and $l=3$ can be obtained with the proposed metasurface. The design process is similar to that introduced previously, and the final phase functions imparted to the transmission wave are shown in the upper panels of Figures 4(a)-4(c) for $l=2$ at $\delta_{r}=0^{\circ}, l=2$ at $\delta_{r}=15^{\circ}$, and $l=3$ at $\delta_{r}=0^{\circ}$, respectively. Clearly, conical radiations were realized with a cone angle tilted in the designed directions, as verified by the $2 \mathrm{D}$ results in the $E$ and $H$ planes. The simulated cone angles for $l=2$ at $\delta_{r}=0^{\circ}, l=2$ at $\delta_{r}=15^{\circ}$, and $l=3$ at $\delta_{r}=0^{\circ}$ were $\alpha=8^{\circ}, \alpha=16^{\circ}$, and $\alpha=11^{\circ}$, respectively. The cone angle can be further flexibly tuned by applying a radial phase gradient to the metasurface, and the 

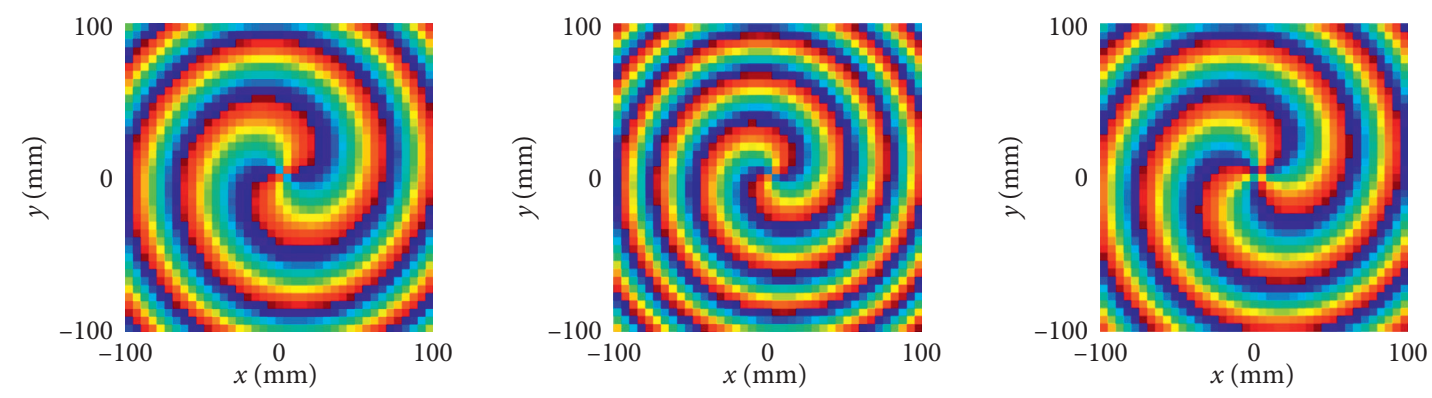

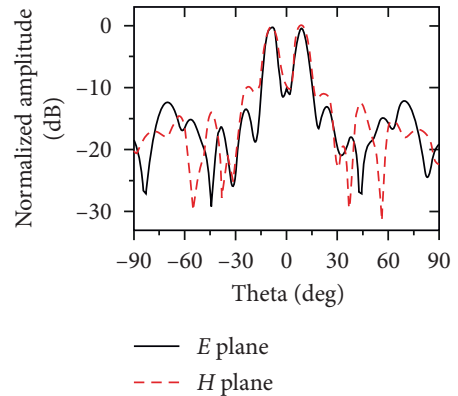

(a)

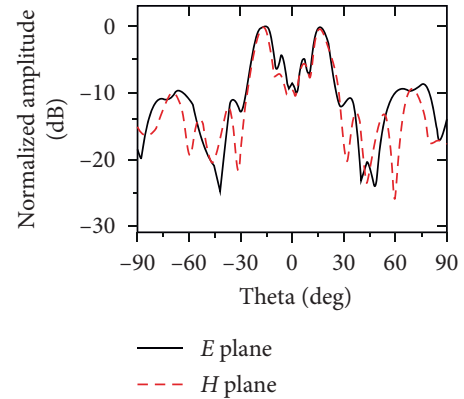

(b)

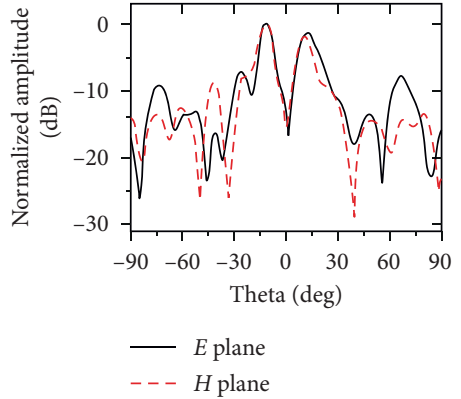

(c)

Figure 4: Results for (a) $l=2$ and $\delta_{r}=0^{\circ}$, (b) $l=2$ and $\delta_{r}=15^{\circ}$, and (c) $l=3$ and $\delta_{r}=0^{\circ}$. The upper panels show the designed phase function of the metasurface for generating a conical beam carrying OAM, and the bottom panels show the simulated $2 \mathrm{D}$ far-field radiation patterns of the metasurface.

OAM mode is not limited to that demonstrated here. In fact, the method can theoretically realize an OAM beam under a larger mode by designing phase distributions on the aperture. However, in ideal OAM beam generation, the elements for different phase responses should be equal, something that cannot be realized by the proposed metasurface because of the square shape and phase discretization when the physical size of the meta-atom is considered. When the OAM mode is very large (e.g., $l>10$ ), the energy distribution of the far-field radiation pattern will be nonuniform in the cone-angle direction. This problem may be mitigated by a metasurface having a circular shape, larger aperture size, and smaller meta-atoms. We also investigated the limits of cone angle tuning for a given OAM mode of $l=1$. As the radial phase gradient increased, not only did the cone angle gradually increase, but the side lobes increased as well. When $\delta_{r}=54^{\circ}$, the cone angle was approximately $42^{\circ}$, whereas the maximum side lobe level was approximately $-3 \mathrm{~dB}$. It should be noted that although the bare radial phase gradient can generate a conical-like beam and achieve flexible control of the cone angle [21], the generated beam intrinsically has a large side lobe at a zenith of $\theta=0^{\circ}$, which will affect the overall performance of the conical beam and limit its realworld application. To overcome this drawback, an OAM mode with a vortex phasefront should be employed, which naturally leads to a singular point in the propagation direction in both the near and far fields. The aforementioned results verified that the proposed metasurface can generate a conical beam through flexible control of its cone angle and the OAM mode.

\section{Experimental Verification}

To demonstrate the design principles experimentally, a sample was fabricated using a standard print board technique and then characterized using a near-field scanning system in a microwave chamber. A photograph of the fabricated metasurface with $l=1$ at $\delta_{r}=0^{\circ}$ is shown in the left panel of Figure 5(a), and an enlarged view of the metasurface is shown in the inset. The metasurface and feed source had the same parameters as those of the simulated model, and the numerical results are shown in the left panel of Figures 3(c) and 3(d). The upper and bottom right panels show the top and bottom views of the fabricated feed source, respectively. In all measurements, the fabricated patch antenna was used as the feed source to illuminate the metasurface for generating the conical beam, which was the same as that used in the simulations. To validate the OAM behavior of the generated beam, the emerging wavefront transmitted from the metasurface was experimentally measured using a 3D near-field scanning system, as shown in Figure 5(b). A dipole probe was used to detect the electric field distribution in the observation plane pixel by pixel. The predefined observation plane ( $x-y$ plane) was set as $500 \mathrm{~mm}$ from the metasurface, and the moving step of the probe was set as $4 \mathrm{~mm}$. The feed source antenna and detecting probe were connected to the two ports of a vector network analyzer (Agilent N5244). Additional details on the measurement setup can be found in our previous work, where we use a schematic to illustrate the field mapping system [13]. The spatial phase distribution of OAM mode $l=1$ can be clearly 


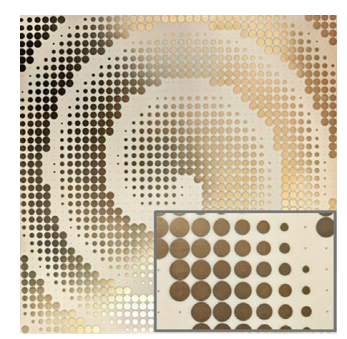

(a)
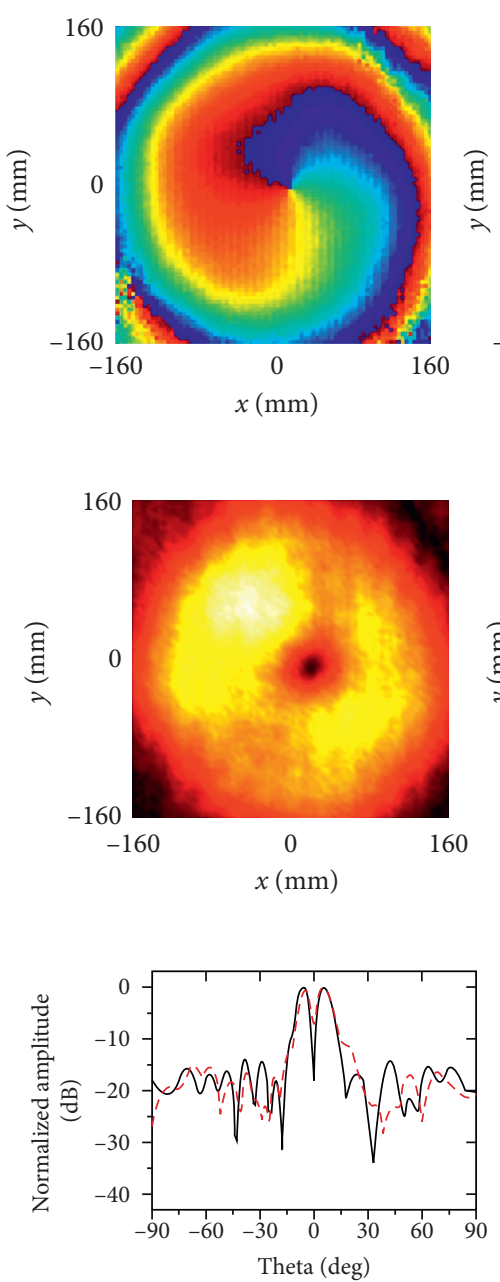

- Sim.
--- Mea.
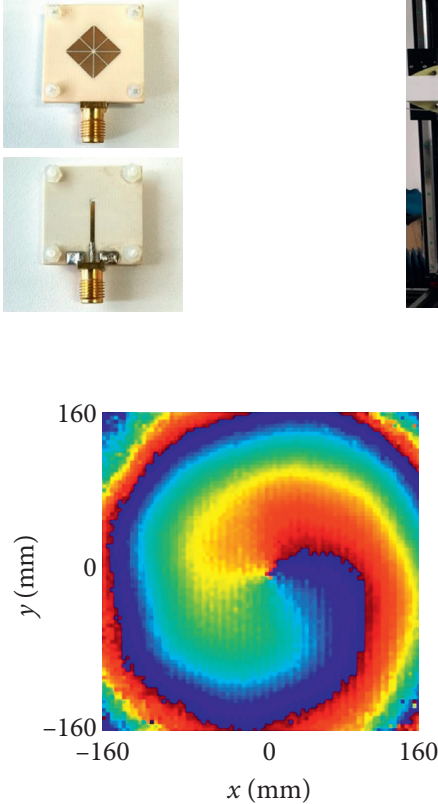

(c)

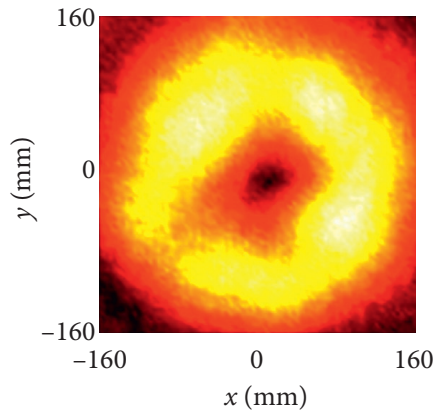

(d)

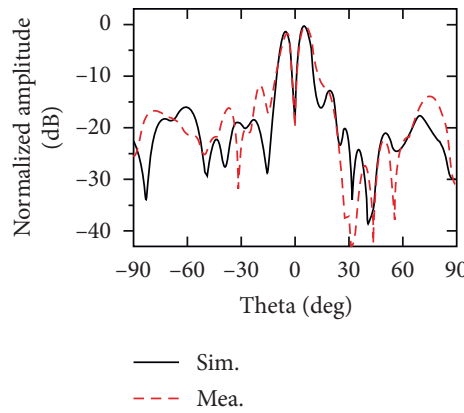

(e)

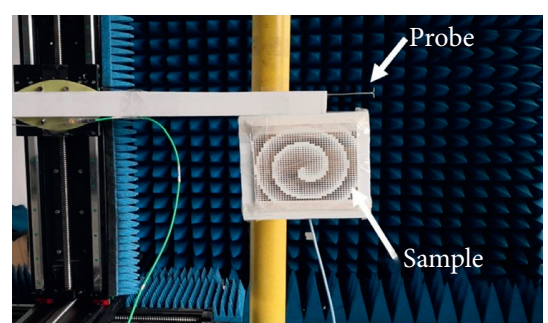

(b)
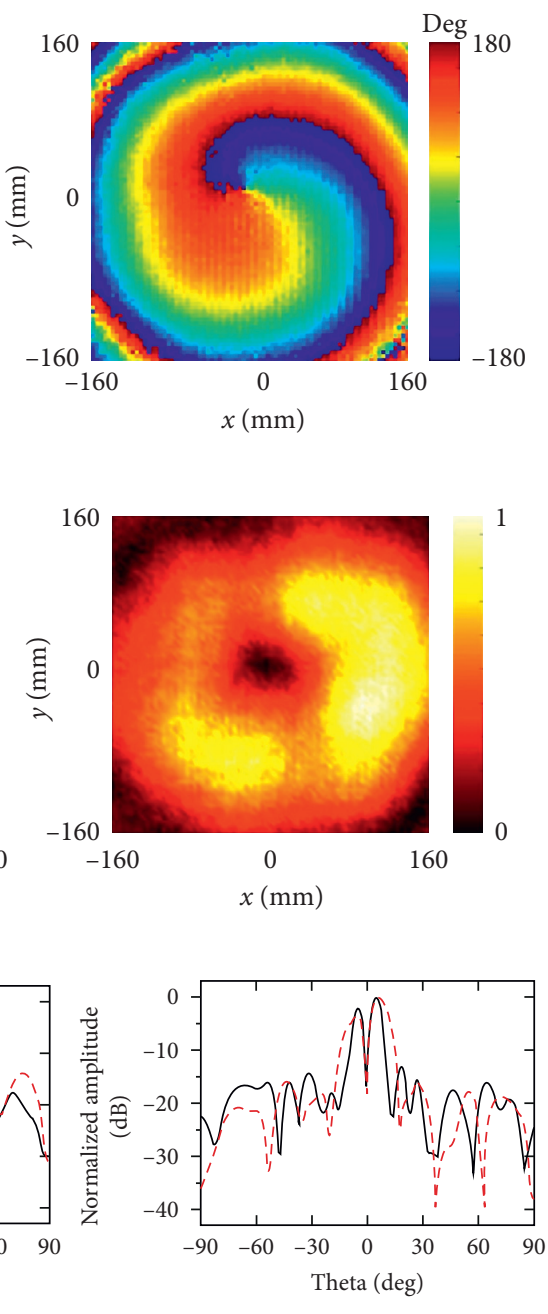

- Sim.
--- Mea.

Figure 5: (a) Photograph of the fabricated metasurface (left panel) and feed source (right panel). (b) Experimental setup for electric field mapping. (c) Measured distribution of the transmission phase. (d) Measured distribution of the transmission amplitude. (e) Measured farfield $2 \mathrm{D}$ radiation pattern at $11.5 \mathrm{GHz}$ (left panels), $13 \mathrm{GHz}$ (middle panels), and $13.5 \mathrm{GHz}$ (right panels).

observed in Figure 5(c) at the different frequencies of 11.5, 13, and $13.5 \mathrm{GHz}$. The amplitude distributions of the OAM beam are shown in Figure 5(d), where a singular point can be observed at the center of the conical beam, verifying its vortex characteristics. The amplitude slightly fluctuated, which was attributed to the nonunitary transmission amplitude and imperfect fabrication and measurement. The measured near-field distribution demonstrated the OAM behavior of the generated beam. Next, the far-field radiation patterns were measured to validate the conical property. In the far-field measurement, a horn antenna was used as the detector placed in the far-field region, and the sample was placed on a turntable that could be freely rotated around its center at an angle step of $0.5^{\circ}$. The receiving antenna was placed approximately $10 \mathrm{~m}$ from the metasurface to consider fully the beam spread effect of the OAM beam and to 

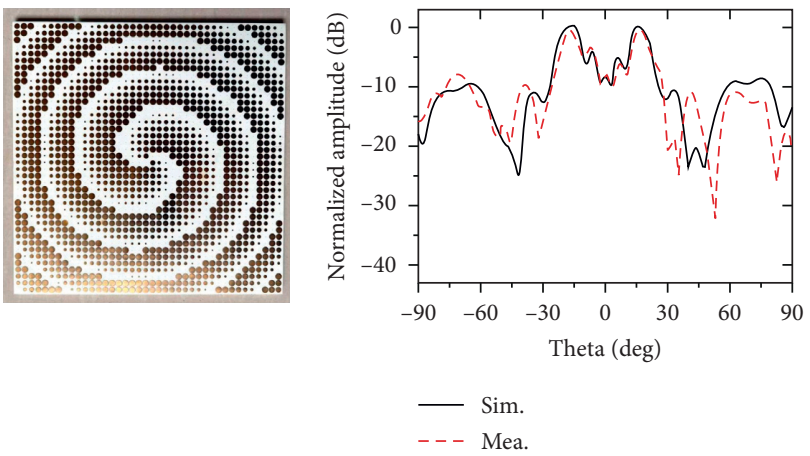

(a)

(b)

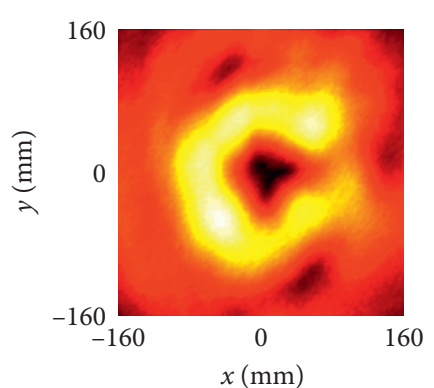

(c)

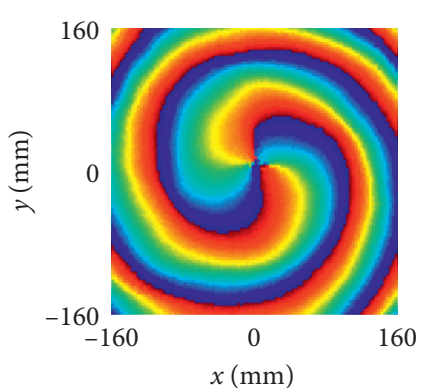

(d)

Figure 6: (a) Photograph of the fabricated metasurface. (b) Measured far-field 2D radiation pattern of the $E$ plane. (c) Measured distribution of the transmission amplitude. (d) Measured distribution of the transmission phase at $13 \mathrm{GHz}$.

measure precisely the far-field radiation pattern. The normalized radiation patterns in the $E$ plane are shown in Figure 4(e), and good agreement could be observed between the measured and simulated results. The measured cone angle was $\alpha=5^{\circ}$, consistent with the simulated cone angle. The cone angle was slightly shifted at these frequencies because of the inherent dispersion of the metasurface. Low side-lobe levels were observed, indicating that most of the emission energy was transformed to its main lobe directions. The amplitude was less than $-10 \mathrm{~dB}$ along the $z$ axis $\left(\theta=0^{\circ}\right)$, verifying the characteristics of the OAM beam. To demonstrate further the ability of the proposed metasurface in controlling the conical beam radiation, we fabricated a sample with a phase profile of OAM mode $l=2$ at $\delta_{r}=15^{\circ}$. A photograph of the sample and measured results are shown in Figure 6. This fabricated metasurface and feed source had the same parameters as those of the simulated model, and the numerical results are shown in the left panel of Figure 4(b). The far-field radiation pattern presented in Figure 6(b) clearly shows the beam spread with the main lobe direction tilted to approximately $17^{\circ}$ in the azimuth angle. We further measured the electric field distribution of the metasurface to verify the OAM mode. The measured amplitude and phase distributions are shown in Figures 6(c) and 6(d), respectively. The measured plane was set as $500 \mathrm{~mm}$ from the metasurface aperture. Low energy distribution was observed in the center of the amplitude distribution, whereas an OAM mode of $l=2$ was observed in the measured phase distribution. Thus, the aforementioned results demonstrated that the transmissive metasurface could flexibly reshape the incidence into desirable conical beam carrying OAM modes. Traditional methods of using antenna arrays to achieve conical beam radiation should employ a complex feeding network to address each antenna of the array individually by a coaxial or transmission line, and the complexity and cost of which are considerably improved if the total aperture size is increased [2-5]. Because the total number of the elements of the array is usually limited, (e.g., approximately 10), the beam width of the simulated or measured conical beam in the far-field region is typically large, and precisely controlling the cone angle is difficult. Compared with these traditional means of generating a conical beam, the proposed metasurface has the advantages of being easily extendable and have subwavelength-sized elements that enable flexible and precise control of the cone angle.

\section{Conclusion}

We proposed a transmissive metasurface with a cascaded structure for control of the conical beam carrying OAM mode. A patch antenna was used as the feed source, and the spherical wavefront of which was reshaped into conical beam radiation by the metasurface with a spatially varying phase distribution. Several design examples of conical beam generation were realized by the proposed metasurface, demonstrating its capability in controlling the conical beam, including the cone angle and OAM mode. We showed that the tuning of the cone angle relied on the diverging effect of the OAM beam, which could be controlled by applying both radial and circumferential phase gradients to the proposed metasurface. Experiments were conducted to validate the full-wave simulation results, and good agreement was observed between them. The proposed metasurface offers a flexible route to generate and control the conical beam in the microwave region. We envision that the proposed method may be extended to generate multiple OAM beams with controllable cone angles with the proper addition of a field multiplexing technique, which may have promising uses in many practical applications such as beam forming, multiple target tracking, and wireless communications. Through the addition of tunable components (e.g., electric-driven diodes), the metasurface can be designed with tunable phase responses that will enable dynamic control of conical beam generation, considerably increasing the impact of the proposed method. Although we experimentally demonstrated only prototypes in the microwave region, the proposed method is not limited in this band and can be readily extended to higher frequencies such as terahertz or even the optical region. This will facilitate the development of novel devices and advanced processing of EM waves. 


\section{Data Availability}

The data used to support the findings of this study are available from the corresponding author upon reasonable request.

\section{Conflicts of Interest}

The authors declare that they have no conflicts of interest.

\section{Acknowledgments}

This work was supported in part by National Key Research and Development Program of China under Grant no. 2017YFA0700201 and National Natural Science Foundation of China (NSFC) under Grant nos. 61801207, 91963128, 62071215, and 61731010 and partially supported by the Priority Academic Program Development of Jiangsu Higher Education Institutions (PAPD) and the Fundamental Research Funds for the Central Universities and Jiangsu Provincial Key Laboratory of Advanced Manipulating Technique of Electromagnetic Wave.

\section{References}

[1] W. H. Chen, J. W. Sun, X. Wang et al., "A novel planar switched parasitic array antenna with steered conical pattern," IEEE Transactions on Antennas and Propagation, vol. 55, no. 6, pp. 1883-1887, 2007.

[2] K. L. Lau and K. M. Luk, "A wideband circularly polarized conical-beam patch antenna," IEEE Transactions on Antennas and Propagation, vol. 54, no. 5, pp. 1591-1594, 2006.

[3] L. Cui, S.-S. Qi, W. Wu, and D.-G. Fang, "High gain conical beam antenna array exploiting grating lobes," IEEE Transactions on Antennas and Propagation, vol. 63, pp. 848-853, 2014.

[4] K.-C. Chen, Y. Qian, C.-K. Tzuang, and T. Itoh, “A periodic microstrip radial antenna array with a conical beam," IEEE Transactions on Antennas and Propagation, vol. 51, pp. 756765, 2003.

[5] S.-H. Son, S.-I. Jeon, C.-J. Kim, and W. Hwang, "GA-based design of multi-ring arrays with omnidirectional conical beam pattern," IEEE Transactions on Antennas and Propagation, vol. 58, pp. 1527-1535, 2010.

[6] N. Yu, P. Genevet, M. A. Kats et al., "Light propagation with phase discontinuities: generalized laws of reflection and refraction,” Science, vol. 334, no. 6054, pp. 333-337, 2011.

[7] S. Sun, Q. He, S. Xiao, Q. Xu, X. Li, and L. Zhou, "Gradientindex meta-surfaces as a bridge linking propagating waves and surface waves," Nature Materials, vol. 11, no. 5, pp. 426-431, 2012.

[8] K. Chen, Y. Feng, F. Monticone et al., "A reconfigurable active Huygens' metalens," Advanced Materials, vol. 29, Article ID 1606422, 2017.

[9] K. Li, Y. Guo, M. Pu et al., "Dispersion controlling meta-lens at visible frequency," Optics Express, vol. 25, no. 18, Article ID 21419, 2017.

[10] Y. Li, J. Zhang, S. Qu et al., "Wideband radar cross section reduction using two-dimensional phase gradient metasurfaces," Applied Physics Letters, vol. 104, no. 22, Article ID 221110, 2014.
[11] G. Zheng, H. Mühlenbernd, M. Kenney, G. Li, T. Zentgraf, and S. Zhang, "Metasurface holograms reaching $80 \%$ efficiency," Nature Nanotechnology, vol. 10, no. 4, pp. 308-312, 2015.

[12] T. J. Cui, L. Li, S. Liu et al., "Information metamaterial systems," iScience, vol. 23, no. 8, Article ID 101403, 2020.

[13] K. Chen, G. Ding, G. Hu et al., Advanced Materials, vol. 32, Article ID 1906352, 2020.

[14] C. Zhang, J. Yang, L. X. Yang et al., "Convolution operations on time-domain digital coding metasurface for beam manipulations of harmonics," Nanophotonics, vol. 9, no. 9, pp. 2771-2781, 2020.

[15] T. Cai, G. Wang, S. Tang et al., "High-efficiency and full-space manipulation of electromagnetic wave fronts with metasurfaces," Physical Review Applied, vol. 8, Article ID 034033, 2017.

[16] N. Zhang, K. Chen, Y. Zheng et al., "Programmable coding metasurface for dual-band independent real-time beam control," IEEE Journal on Emerging and Selected Topics in Circuits and Systems, vol. 10, no. 1, pp. 20-28, 2020.

[17] F. Monticone, N. M. Estakhri, and A. Alù, "Full control of nanoscale optical transmission with a composite metascreen," Physical Review Letters, vol. 110, Article ID 203903, 2013.

[18] J. Wang, Y. Li, Z. H. Jiang et al., "Metantenna: when metasurface meets antenna again," IEEE Transactions on Antennas and Propagation, vol. 68, no. 3, pp. 1332-1347, 2020.

[19] A. M. Yao and M. J. Padgett, "Orbital angular momentum: origins, behavior and applications," Advances in Optics and Photonics, vol. 3, no. 2, p. 161, 2011.

[20] G. Ding, K. Chen, X. Luo, J. Zhao, T. Jiang, and Y. Feng, "Dual-helicity decoupled coding metasurface for independent spin-to-orbital angular momentum conversion," Physical Review Applied, vol. 11, Article ID 44043, 2019.

[21] G. Ding, K. Chen, T. Jiang, B. Sima, J. Zhao, and Y. Feng, "Full control of conical beam carrying orbital angular momentum by reflective metasurface," Optics Express, vol. 26, no. 16, Article ID 20990, 2018.

[22] G. Li, M. Kang, S. Chen et al., "Spin-enabled plasmonic metasurfaces for manipulating orbital angular momentum of light," Nano Letters, vol. 13, no. 9, pp. 4148-4151, 2013.

[23] Y. Li, X. Li, L. Chen et al., "Orbital angular momentum multiplexing and demultiplexing by a single metasurface," Advanced Optical Materials, vol. 5, no. 2, Article ID 1600502, 2017.

[24] H. Sroor, Y.-W. Huang, B. Sephton et al., "High-purity orbital angular momentum states from a visible metasurface laser," Nature Photonics, vol. 14, no. 8, pp. 498-503, 2020.

[25] K. Zhang, Y. Yuan, X. Ding et al., "Polarization-engineered noninterleaved metasurface for integer and fractional orbital angular momentum multiplexing," Laser and Photonics Reviews, vol. 15, no. 1, Article ID 2000351, 2021.

[26] E. Karimi, S. A. Schulz, I. De Leon, H. Qassim, J. Upham, and R. W. Boyd, "Generating optical orbital angular momentum at visible wavelengths using a plasmonic metasurface," Light Science and Applications, vol. 3, p. e167, 2014.

[27] Y. Yuan, K. Zhang, B. Ratni et al., "Independent phase modulation for quadruplex polarization channels enabled by chirality-assisted geometric-phase metasurfaces," Nature Communications.vol. 11, p. 4186, 2020.

[28] O. Edfors and A. J. Johansson, "Is orbital angular momentum (OAM) based radio communication an unexploited area?" IEEE Transactions on Antennas and Propagation, vol. 60, no. 2, pp. 1126-1131, 2012.

[29] A. F. Morabito, L. Di Donato, and T. Isernia, "Orbital angular momentum antennas: understanding actual possibilities 
through the aperture antennas theory," IEEE Antennas and Propagation Magazine, vol. 60, no. 2, pp. 59-67, 2018.

[30] M. Barbuto, M.-A. Miri, A. Alù, F. Bilotti, and A. Toscano, "A topological design tool for the synthesis of antenna radiation patterns," IEEE Transactions on Antennas and Propagation, vol. 68, no. 3, pp. 1851-1859, 2020.

[31] J. Wang, K. Liu, Y. Cheng, and H. Wang, "Vortex SAR imaging method based on OAM beams design," IEEE Sensors Journal, vol. 19, no. 24, pp. 11873-11879, 2019.

[32] K. Liu, X. Li, Y. Gao, H. Wang, and Y. Cheng, "Microwave imaging of spinning object using orbital angular momentum," Journal of Applied Physics, vol. 122, no. 12, Article ID 124903, 2017.

[33] S. M. Mohammadi, L. K. S. Daldorff, J. E. S. Bergman et al., "Orbital angular momentum in radio-A system study," IEEE Transactions on Antennas and Propagation, vol. 58, no. 2, pp. 565-572, 2010.

[34] C. Pfeiffer and A. Grbic, "Metamaterial Huygens' surfaces: tailoring wave fronts with reflectionless sheets," Physical Review Letters, vol. 110, Article ID 197401, 2013.

[35] A. Epstein, J. P. S. Wong, and G. V. Eleftheriades, "Cavityexcited Huygens' metasurface antennas for near-unity aperture illumination efficiency from arbitrarily large apertures," Nature Communications, vol. 7, p. 1, 2016.

[36] C. Pfeiffer and A. Grbic, "Cascaded metasurfaces for complete phase and polarization control," Applied Physics Letters, vol. 102, no. 23, Article ID 231116, 2013.

[37] Q. He, S. Sun, S. Xiao, and L. Zhou, "High-efficiency metasurfaces: principles, realizations, and applications," Advanced Optical Materials, vol. 6, no. 19, Article ID 1800415, 2018. 